DOI: $10.2478 / \mathrm{adms}-2020-0019$

\author{
Sudipta Sen*, N. B. Manik \\ Condensed Matter Physics Research Centre, Department of Physics, Jadavpur University, \\ Kolkata-700032, India \\ *sagnike000@gmail.com
}

\title{
EFFECT OF DIFFERENT SIZED MULTI WALLED CARBON NANOTUBES ON THE BARRIER POTENTIAL AND TRAP CONCENTRATION OF MALACHITE GREEN DYE BASED ORGANIC DEVICE
}

\begin{abstract}
Present work shows effect of $8 \mathrm{~nm}$ diameter and $30 \mathrm{~nm}$ diameter multi walled carbon nanotubes (MWCNT) on the barrier potential and trap concentration of Malachite Green (MG) dye based organic device. MWCNTs are basically a bundle of concentric single-walled carbon nanotubes with different diameters. In this work, ITO coated glass substrate and aluminium have been used as front electrode and back electrode respectively and the spin coating method is used to prepare the MG dye based organic device. It has been observed that both barrier potential and trap concentration are in correlation. Estimation of both these parameters has been done from current-voltage characteristics of the device to estimate the trap energy and the barrier potential of the device. Device turn-on voltage or the transition voltage is also calculated by using current-voltage characteristics. In presence of $8 \mathrm{~nm}$ diameter MWCNT, the transition voltage is reduced from $3.9 \mathrm{~V}$ to $2.37 \mathrm{~V}$, the barrier potential is lowered to $0.97 \mathrm{eV}$ from $1.12 \mathrm{eV}$ and the trap energy is lowered to $0.028 \mathrm{eV}$ from $0.046 \mathrm{eV}$ whereas incorporation of $30 \mathrm{~nm}$ diameter MWCNT shows reduction of transition voltage from $3.9 \mathrm{~V}$ to $2.71 \mathrm{~V}$ and a reduction of barrier potential and trap concentration from $1.12 \mathrm{eV}$ to $1.03 \mathrm{eV}$ and from $0.046 \mathrm{eV}$ to $0.035 \mathrm{eV}$ respectively. Presence of both $8 \mathrm{~nm}$ diameter and $30 \mathrm{~nm}$ diameter MWCNT lowers trap energy approximately to 39\% and 24\% respectively and lowers barrier potential approximately to $13 \%$ and $8 \%$ respectively. Estimation of barrier potential is also done by Norde method which shows lowering of the value from $0.88 \mathrm{eV}$ to $0.79 \mathrm{eV}$ and from $0.88 \mathrm{eV}$ to $0.84 \mathrm{eV}$ in presence of both $8 \mathrm{~nm}$ and $30 \mathrm{~nm}$ diameter multi walled carbon nanotubes respectively. Calculation of barrier potential from both the I-V characteristics and Norde method are in unison with each other. Indication of enhancement of charge flow in the device can be ascribed to the truncated values of barrier potential and trap energy.
\end{abstract}

Keywords: Barrier Potential; Malachite Green Dye; MWCNT; Transition Voltage; Trap Energy

\section{INTRODUCTION}

Creation of metal - organic interface and its associated properties profoundly impact the performance of any organic electronic devices [1]. Amorphous nature of organic materials causes 
the formation of highly localized trap states and when these materials come in metal contact, these traps affect the charge flow at metal - organic contact. Process of charge flowing is very much relied on barrier potential at interface. High trap concentration at metal - organic junction leads to lowering of charge flow as the traps act like recombination centres, so, very few mobile charges will be there to overcome the high barrier potential at metal - organic contact. On the other hand, the high barrier potential at the metal - organic contact also hinders the charge injection process, resulting in lower conductivity [2-5]. High interfacial barrier potential also causes the transition voltage of the organic dye based devices to be high. Transition voltage means the voltage at which the organic dye based device starts to conduct. So, both these trap concentration and barrier potential at metal - organic contact have significant impact on charge injection and collection process and effect of both these parameters are required to be reduced considerably so that the device can work optimally. Still, it remains a challenge to achieve reduction of both high barrier potential $\left(\phi_{\mathrm{b}}\right)$ and high trap concentration $\left(\mathrm{E}_{\mathrm{t}}\right)$ at metal - organic contact.

In our work, Malachite Green (MG) dye has been chosen as organic material and it has been sandwiched between two metal electrodes of different work functions namely, Indium Tin Oxide (ITO) and Aluminium (Al). ITO has work function of $4.8 \mathrm{eV}$ and Aluminium has work function of $4.2 \mathrm{eV}$ [6]. We have also incorporated Multiwalled Carbon nanotubes (MWCNT) within the prepared organic device. MWCNTs have several single walled carbon nanotubes in concentric cylinders. It possesses excellent electrical, mechanical and thermal properties and for different organic dye based devices, MWCNT has been used for waste water treatment by removing different organic dyes from aqueous solutions [7-10].Generally, presence of different sized MWCNT shifts the bands within a depth of only a few nanometers from the junction causes decrease in barrier potential and reduces trap concentration [11]. MWCNT incorporation also acts as filler and its high aspect ratio helps in providing easy path for charge percolation due to which the current flow in the device also increases [12].

In present scenario, there is no significant work regarding the analytical relation between barrier potential and trap concentration and the main aim of the present study is to establish an interrelationship between barrier potential and trap concentration of the organic device as both these parameters play a pivotal role regarding the charge injection process of the device. Trap concentration is required to be minimized to lessen the recombination effect. Lowering of recombination effect will allow greater amount of mobile charges to overcome the high barrier potential at metal - organic contact. When the charge flow at metal - organic contact gets increased, the device performance will also be improved in terms of conductivity and efficiency. To reduce both these parameters for device performance enhancement, Multi walled Carbon Nanotubes (MWCNT) within the device has been incorporated and its effect is observed on both of these parameters. Analysis of these two parameters in presence and absence of MWCNT has been mostly done by using the device's I-V plot. Transition voltage is also calculated from same I-V plot. Perusal of the current flow has been done by Richardson - Schottky (RS) model [1314]. We have also used Norde method to estimate barrier potential to check the consistency of the value obtained from device's I-V plot. 


\section{MATERIALS AND SAMPLE PREPARATION}

As mentioned earlier, MWCNTs are special form of carbon nanotubes in which multiple single-walled carbon nanotubes are nested inside one another. MWCNTs have thermal conductivity which is in the range of $3000 \mathrm{~W} / \mathrm{m}-\mathrm{K}$ and have excellent electrochemical properties [15].CAS number of MWCNT is 308068-56-6. We have used two different sized MWCNT, one is $8 \mathrm{~nm}$ diameter MWCNT and the other is $30 \mathrm{~nm}$ diameter MWCNT. Carbon content of both the MWCNTs is of more than $99 \%$. Malachite green is a triphenyl methane dye and cationic dye used for coloring cotton, wool, silk, paper, leather, etc. It is also used as parasiticide, fungicide, antiprotozoan, and antibacterial agent [16]. CAS number and colour index number of Malachite Green dye are 2437-29-8 and 42000 respectively. Molecular weight of Malachite Green dye is 463.50 and its empirical formula is $\left(\mathrm{C}_{23} \mathrm{H}_{25} \mathrm{~N}_{2}\right)_{2} 3 \mathrm{C}_{2} \mathrm{H}_{2} \mathrm{O}_{4}$. Both MWCNT and Malachite Green dye are bought from Sigma- Aldrich, India. Fig. 1 (a) and Fig. 1(b) represent schematic structures of MG dye and MWCNT respectively. Fig. 1(c) and Fig. 1(d) represent raw material form of both MG dye and MWCNT respectively. In this work, we have also used Poly Methyl Methacrylate (PMMA). PMMA is obtained from Merck Specialties Pvt. Ltd, Mumbai. PMMA solution acts as an inert binder [17]. In our work, PMMA solution has been prepared by mixing $10 \mathrm{ml}$ distilled water with1mg Poly Methyl Methacrylate (PMMA). Two electrodes which we have used in our experiment are Indium Tin Oxide (ITO) coated glass substrate and Aluminum respectively. Commercial grade of aluminium used as the electrode is Grade 1050.

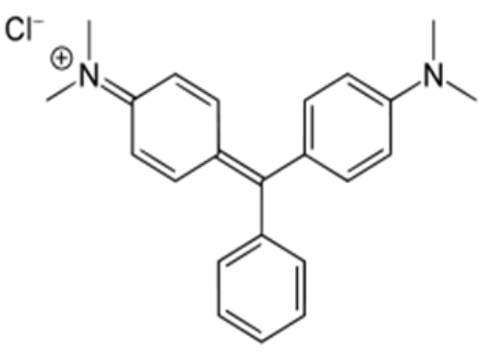

(a)

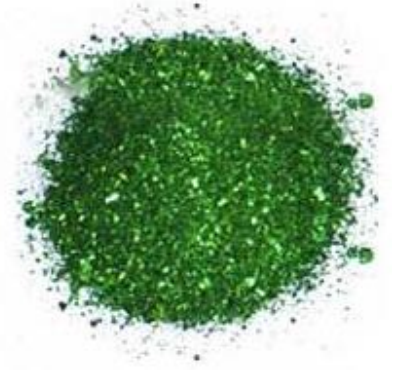

(c)

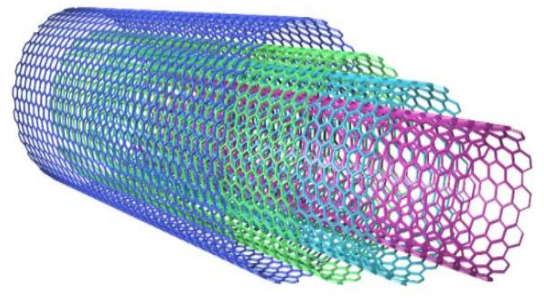

(b)

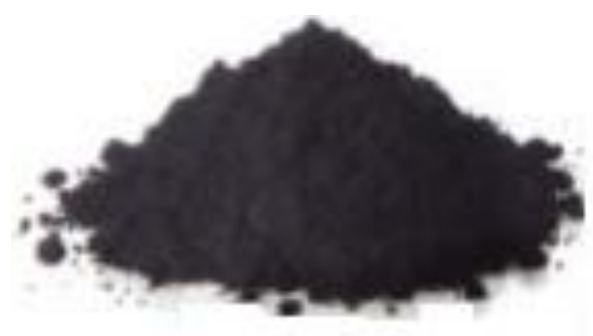

(d)

Fig. 1. Schematic structures of (a) MG and (b) MWCNT and in Raw material form (c) MG and (d) MWCNT

In one of our earlier works [18], we have mentioned the process of making the solution of this MG dye and MWCNT. In PMMA solution, $1 \mathrm{mg}$ of MG dye is added and stirred for $30 \mathrm{~min}$. 
The dye solution is then divided into three parts in three pre-cleaned test tubes. In one test tube, there is only MG dye solution without any presence of MWCNT and in the other two test-tubes, MWCNT of $8 \mathrm{~nm}$ diameter and MWCNT of $30 \mathrm{~nm}$ diameter are added respectively and stirred for 3 hours to get two homogeneous solutions. One is the solution of MG dye and $8 \mathrm{~nm}$ diameter MWCNT and the other is the solution of MG dye and $30 \mathrm{~nm}$ diameter MWCNT.

To prepare only MG dye based organic device, MG dye solution is spin coated with a speed of $1500 \mathrm{rpm}$ and then dried at $3500 \mathrm{rpm}$ speed on an ITO coated glass substrate. The same solution is similarly spin coated on Al electrode. These electrodes are attached together in semidry state in forming only MG dye based organic device. Similarly, other two MG dye based organic devices in presence of $8 \mathrm{~nm}$ MWCNT and $30 \mathrm{~nm}$ MWCNT respectively have also been prepared. All these three cells are dried in vacuum desiccators at room temperature for 12 hours before characterization. Representations of prepared devices are depicted in Fig. 2. In Fig. 2, the thickness of each layer of the sandwiched structured device which is comprised of ITO electrode, MG dye- nanoparticles composite and $\mathrm{Al}$ electrode are $1.1 \mu \mathrm{m}, 3 \mu \mathrm{m}$ and $1.5 \mu \mathrm{m}$ respectively.

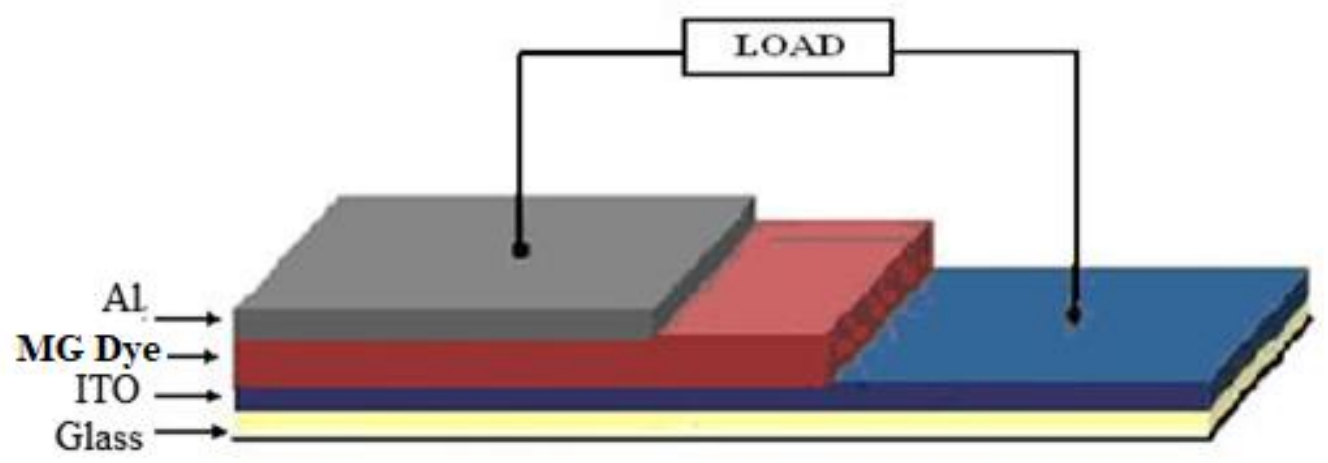

Fig. 2. Sandwich structure of organic device

\section{MEASUREMENTS}

The current - voltage (I-V) measurement using Keithley 2400 source measure unit has been done in this work. Same technique is followed as mentioned in one of our earlier works [19]. The voltage range is between 0 to $5 \mathrm{~V}$ in steps of $0.2 \mathrm{~V}$ with delay of $500 \mathrm{~ms}$. The room temperature was kept at $26^{\circ} \mathrm{C}$.

\section{RESULTS AND DISCUSSION}

The charge carriers need to overcome the barrier potential at the metal - organic layer interface, so that current can flow at the interface. To overcome the barrier potential, these charge carriers require sufficient energy which can be explained by the Richardson - Schottky (R-S) thermionic emission theory. The essential assumption of this theory is that once an electron from metal has acquired sufficient thermal energy, it can be injected into the organic layer by overcoming the barrier potential at metal - organic layer interface and this charge injection will 
result in interfacial current flow [20].The interfacial current (I) as per Richardson - Schottky(RS) thermionic emission theory is:

$\mathrm{I}=\mathrm{I}_{\mathrm{S}}\left(\exp \left(\frac{\mathrm{qV}}{\mathrm{nkT}}\right)-1\right)$

$\mathrm{I}_{\mathrm{S}}=A A^{*} \mathrm{~T}^{2} \exp \left(-\frac{\mathrm{q} \phi_{\mathrm{b}}}{\mathrm{kT}}\right)$

$\mathrm{q}$ is the charge of electron, $\mathrm{V}$ is the applied voltage, $\mathrm{A}$ is the device area, $\mathrm{k}$ is the Boltzmann's constant, $\mathrm{T}$ is absolute temperature, $\mathrm{A}^{*}$ is the effective Richardson constant, $\mathrm{n}$ is the ideality factor and $\phi_{b}$ is the barrier potential and $I_{S}$ is the saturation current [21-27].

The I-V plot of organic device in absence of any nanoparticle and under the influence of 8 $\mathrm{nm}$ MWCNT and $30 \mathrm{~nm}$ MWCNT have been depicted in Fig. 3. From the Fig. 3, it can be observed that impact of MWCNTs increase charge flow. With $8 \mathrm{~nm}$ MWCNT and $30 \mathrm{~nm}$ MWCNT, the current has been increased three times and two times respectively compared to without MWCNT in the device.

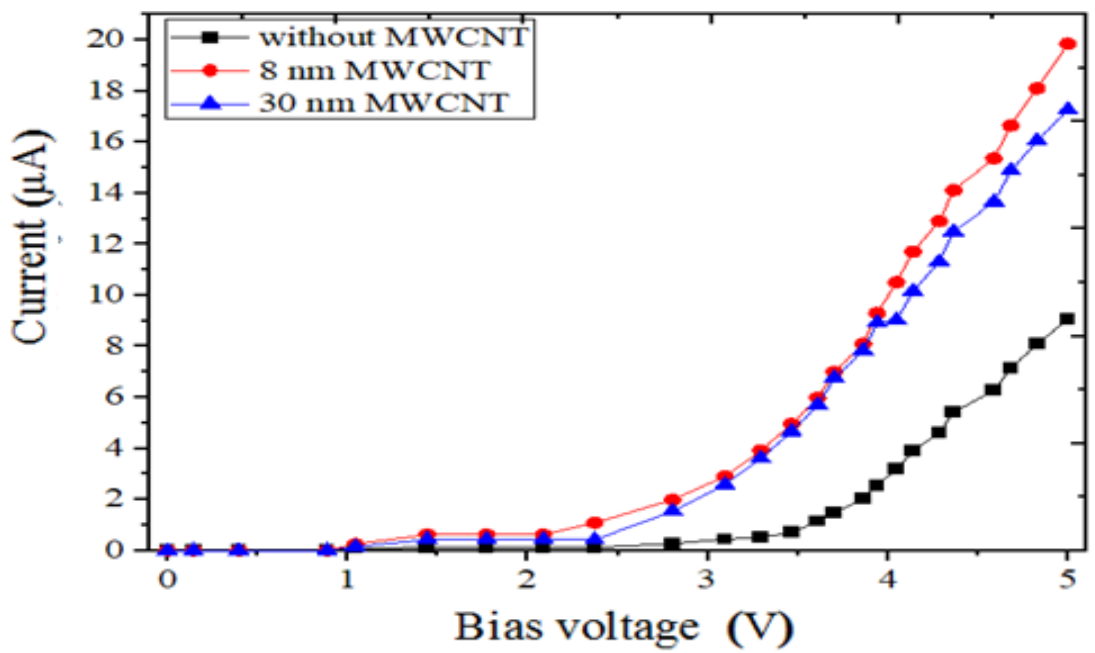

Fig. 3. Current-voltage plot of organic device without any nanoparticle and with $8 \mathrm{~nm}$ and $30 \mathrm{~nm}$ MWCNT respectively

The barrier potential can be deduced from equation (2) as:

$\phi_{\mathrm{b}}=\frac{\mathrm{kT}}{\mathrm{q}} \ln \left(\frac{\mathrm{AA}^{*} \mathrm{~T}^{2}}{\mathrm{I}_{\mathrm{S}}}\right)$

Barrier potential has been calculated by semi log I-V plot of organic device without and with $8 \mathrm{~nm}$ MWCNT and $30 \mathrm{~nm}$ MWCNT in Fig. 4. Intercept with voltage axis gives the value of saturation current and by using equation (3), barrier potential has been estimated. 


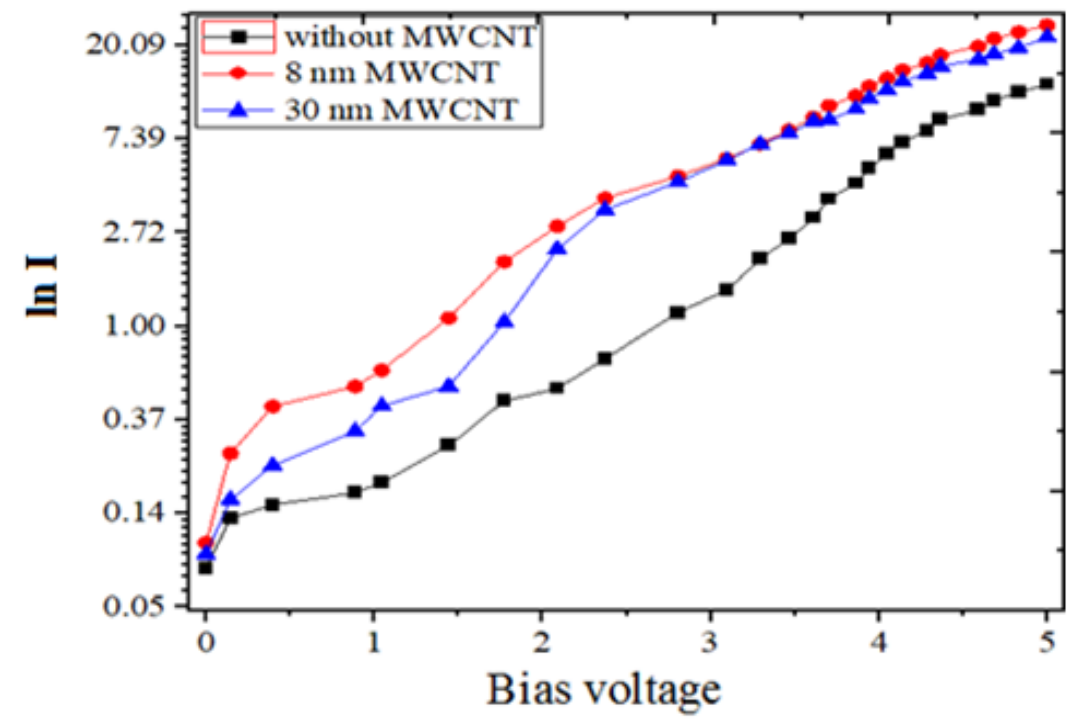

Fig. 4. In I-V plot of organic device without any nanoparticle and with $8 \mathrm{~nm}$ and $30 \mathrm{~nm}$ MWCNT respectively

Measurement of barrier potential has been done by Norde function which shows interrelationship between the function $\mathrm{f}(\mathrm{v})$ and current $\mathrm{i}(\mathrm{v})$ which has been given in the equation (4) [28-29].

$f(v)=\left(\frac{v}{y}\right)-\frac{k T}{q} \ln \left(\frac{i(v)}{{A A^{*} T^{2}}^{2}}\right)$

Where, $y$ is the first integer greater than $\mathrm{n}$. The value of current $\mathrm{i}\left(\mathrm{v}_{0}\right)$ corresponding to minimum value of Norde's function $\mathrm{f}\left(\mathrm{v}_{0}\right)$, where $\mathrm{v}_{0}$ is the minimum voltage.

Taking derivative of $\mathrm{f}(\mathrm{v})$ and equating it to 0 will give the current $\mathrm{i}_{0}$ at the minimum point of $\mathrm{f}(\mathrm{v})$. The minimum voltage $\mathrm{v}_{0}$ is obtained as:

$\mathrm{v}_{0}=\frac{\mathrm{kT}}{\mathrm{q}}+\ln \left(\frac{\mathrm{i}_{0}}{\mathrm{AA}^{*} \mathrm{~T}^{2}}\right)$

and the minimum value of $\mathrm{f}(\mathrm{v})$ becomes

$f\left(v_{0}\right)=\frac{v_{0}}{y}-\frac{k T}{q}\left(\frac{i_{0}}{A^{*} T^{2}}\right)$ 
Estimation of barrier potential is done by equation (7) obtained from Norde function, which is shown in Fig. 5 without and with $8 \mathrm{~nm}$ diameter and $30 \mathrm{~nm}$ diameter MWCNT respectively [30-31].

$\phi_{\mathrm{b}}=\mathrm{f}\left(\mathrm{v}_{0}\right)+\frac{\mathrm{v}_{0}}{y}-\frac{\mathrm{kT}}{\mathrm{q}}$

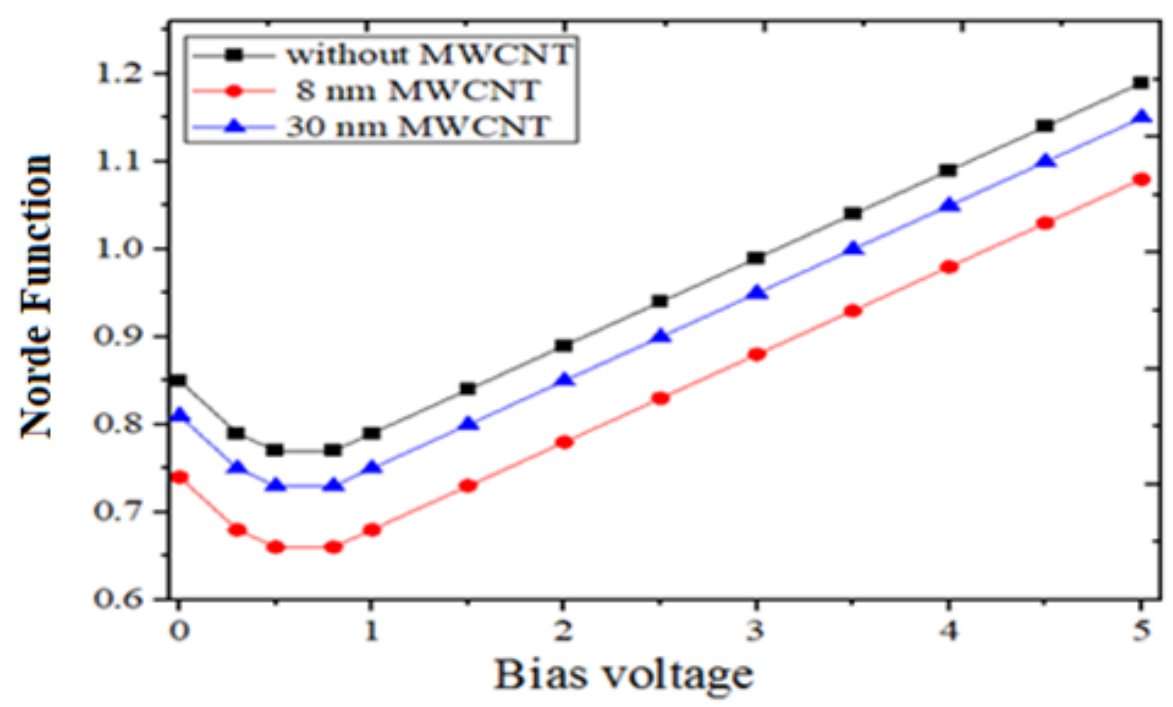

Fig. 5. Norde function plot of organic device without any nanoparticle and with $8 \mathrm{~nm}$ and $30 \mathrm{~nm}$ MWCNT respectively

Norde method is applicable for the applied voltages of $(\mathrm{V}>3 \mathrm{kT} / \mathrm{q})$. This method also observes lowering of barrier potential in presence of both the MWCNTs as it has been estimated from I-V characteristics of the device. The small differences in barrier potential obtained by both these methods are due to the different processes of extraction from different regions.

Concentration of traps at metal - organic interface plays a pivotal role in charge injection process of the device. Fig. 6 shows the $\ln \mathrm{I}-\ln \mathrm{V}$ plot of the organic device, without any nanoparticle and with $8 \mathrm{~nm}$ and $30 \mathrm{~nm}$ MWCNT respectively.

The trap energy can be written as expressed in the following equation (8):

$\mathrm{E}_{\mathrm{t}}=\mathrm{mkT}$

Where $\mathrm{E}_{\mathrm{t}}=$ trap energy, $\mathrm{m}=\mathrm{T}_{\mathrm{c}} / \mathrm{T}$, where, $\mathrm{T}_{\mathrm{c}}$ is a characteristic temperature that describe the trap distribution and $\mathrm{T}$ is absolute temperature [32].

From Fig. 6, ' $m$ ' has been calculated and by using equation (8), the trap energy has been estimated without and with $8 \mathrm{~nm}$ diameter and $30 \mathrm{~nm}$ diameter MWCNT of MG dye based organic device. 
Comparing equation (3) and equation (8), it can be inferred that barrier potential and trap energy are proportional to each other, considering other parameters remain unchanged. So, when the concentration of traps reduces, the barrier potential also decreases. So, the interconnection between barrier potential and trap energy can be established analytically.

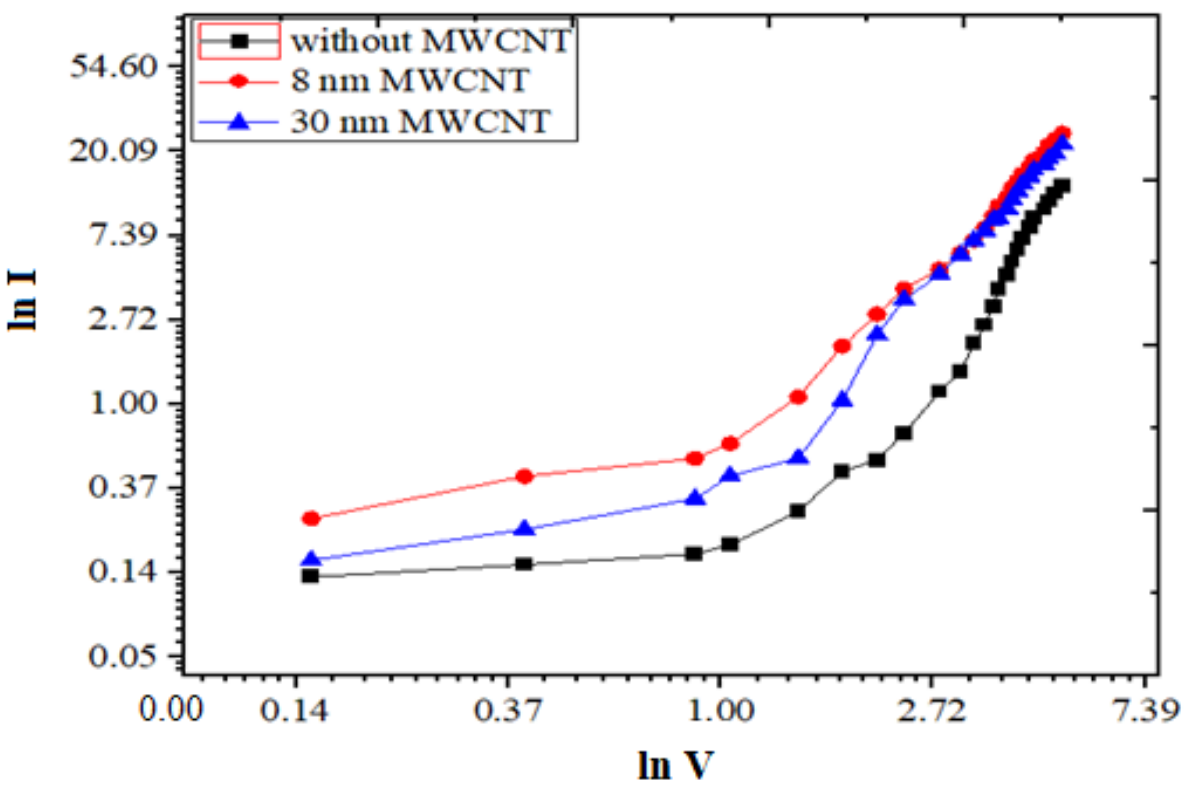

Fig. 6. InI-InV plot of organic device without any nanoparticle and with $8 \mathrm{~nm}$ and $30 \mathrm{~nm}$ MWCNT respectively

The values of transition voltage, trap energy and barrier potential of organic devices without and with $8 \mathrm{~nm}$ and $30 \mathrm{~nm}$ MWCNT are shown in Table 1.

Table 1. Calculation of transition voltage, trap energy and barrier potential of organic devices without and with $8 \mathrm{~nm}$ and $30 \mathrm{~nm}$ MWCNT

\begin{tabular}{cccccc}
\hline Device & $\begin{array}{c}\text { Transition } \\
\text { voltage } \\
(\mathrm{V})\end{array}$ & $\begin{array}{c}\text { Value } \\
\text { of } \mathrm{m}\end{array}$ & $\begin{array}{c}\text { Trap } \\
\text { Energy } \\
(\mathrm{eV})\end{array}$ & $\begin{array}{c}\text { Barrier } \\
\text { potential } \\
\text { from I-V plot } \\
(\mathrm{eV})\end{array}$ & $\begin{array}{c}\text { Barrier potential } \\
\text { using Norde } \\
\text { Function } \\
(\mathrm{eV})\end{array}$ \\
\hline ITO/MG/Al & 3.9 & 1.77 & 0.046 & 1.12 & 0.88 \\
ITO/MG+8nm MWCNT/Al & 2.37 & 1.08 & 0.028 & 0.97 & 0.79 \\
ITO/MG+30nmMWCNT/Al & 2.71 & 1.34 & 0.035 & 1.03 & 0.84 \\
\hline
\end{tabular}

Table 1 shows that $8 \mathrm{~nm}$ diameter and $30 \mathrm{~nm}$ diameter MWCNT decrease trap energy approximately to $39 \%$ and $24 \%$ respectively and they reduce barrier potential approximately to $13 \%$ and $8 \%$ respectively. These results are obtained from I-V characteristics of the device. Norde method also shows $8 \mathrm{~nm}$ diameter and $30 \mathrm{~nm}$ diameter MWCNT lessen the value of barrier potential approximately to $10 \%$ and 5\% respectively. Lessening of transition voltage in presence of both MWCNT allows the device to be turned on at lower voltages. 


\section{CONCLUSIONS}

Influence of both the $8 \mathrm{~nm}$ diameter and $30 \mathrm{~nm}$ diameter MWCNT on barrier potential and trap energy of organic devices has been observed. Barrier potential and trap energy for organic devices under the influence of MWCNTs are calculated by I-V plot. Barrier potential is also estimated by using Norde method. Both the methods remain in unison. We have also shown that barrier potential and trap energy are analytically proportional to each other which means reduction of one parameter will result in lowering of another parameter. Barrier potential and trap energy at metal - organic contact have been reduced under the influence of both $8 \mathrm{~nm}$ and $30 \mathrm{~nm}$ diameter MWCNT. MWCNT also reduces the transition voltage or the turn on voltage of the device which can be ascribed to improved charge flow. It is also observed that the device performance in terms of barrier potential and the trap energy, $30 \mathrm{~nm}$ diameter MWCNT reduces both barrier potential and the trap energy to $8 \%$ and $24 \%$ respectively whereas $8 \mathrm{~nm}$ diameter MWCNT reduces both the parameters to $13 \%$ and $39 \%$ respectively. Due to their smaller sizes and high aspect ratios, $8 \mathrm{~nm}$ diameter MWCNT enhances the charge separation and relaxation process which results in the better performance in terms of the charge injection process of the organic device compared to that of $30 \mathrm{~nm}$ diameter MWCNT. This work will be informative as transition voltage lowering of the device will cause consumed power to decrease and also the charge injection process gets better in terms of barrier potential lowering and trap energy reduction, results in improved device conductivity. The desired level of conductivity can be achieved with much lower loadings.

\section{ACKNOWLEDGEMENTS}

Financial support under Grant No.3482/ (NET-JULY2016) from UGC, India are gratefully acknowledged by Sudipta Sen.

\section{REFERENCES}

1. Liu Y- F., Feng J., Bi Y-G., Yin D., Sun H. B.: Recent Developments in Flexible Organic LightEmitting Devices. Advanced Materials Technologies 4 (2019) 1800371-1-1800371-19.

2. Oehzelt M., Koch N., Heimel G.: Organic semiconductor density of states controls the energy level alignment at electrode interfaces. Nature Communications 5 (2014) 1-8.

3. Haneef H. F., Zeidell A. M., Jurchescu O. D.: Charge carrier traps in organic semiconductors: a review on the underlying physics and impact on electronic devices. Journal of Materials Chemistry C 8 (2020) 759787.

4. Bullejos P. L., Tejada J. A. J., Deen M. J., Marinov O., Datars W. R.: Unified model for the injection and transport of charge in organic diodes. Journal of Applied Physics 103 (2008) 064504-1 -06450412.

5. Armbrust N., Schiller F., Güdde J., Höfer U.: Model potential for the description of metal/organic interface states. Scientific Reports 7 (2017) 1-8. 
6. Kumar S., Iyer S. S. K.: Metal-organic semiconductor interfacial barrier height determination from internal photoemission signal in spectral response measurements. Journal of Applied Physics 121 (2017) 143104-1 -143104-6.

7. Lian Z., Wei C., Gao B., Yang X., Chan Y., et al.: Synergetic treatment of dye contaminated wastewater using microparticles functionalized with carbon nanotubes/titanium dioxide nanocomposites. RSC Advances 10 (2020) 9210-9225.

8. Park J. G., Cheng Q., Lu J., Bao J., Li S., et al.: Thermal conductivity of MWCNT/epoxy composites: The effects of length, alignment and functionalization. Carbon 50 (2012) 2083-2090.

9. Kumanek B., Janas D.: Thermal conductivity of carbon nanotube networks: a review. Journal of Materials Science 54 (2019) 7397-7427.

10. Yang M., Li X., Wang W., Zhang S., Han R.: Adsorption of methyl blue from solution by carboxylic multi-walled carbon nanotubes in batch mode. Desalination and Water Treatment 159 (2019) 365376.

11. Svensson J., Campbell E. E. B.: Schottky barriers in carbon nanotube-metal contacts. Journal of Applied Physics 110 (2011) 111101-1-111101-16.

12. Guo J., Liu Y.,1 Prada-Silvy R., Tan Y., Azad S., Krause B., Potschke P., Grady B. P.: Aspect Ratio Effects of Multi-walled Carbon Nanotubes on Electrical, Mechanical, and Thermal Properties of Polycarbonate/MWCNT Composites. Journal of Polymer Science Part B Polymer Physics 52 (2014) 73-83.

13. Sze S.M., Ng K.K.: Physics of Semiconductor Devices. [3rd ed.], Wiley, New York, 2007.

14. Kumatani A., Li Y., Darmawan P., Minari T., Tsukagoshi K.: On Practical Charge Injection at the Metal/Organic Semiconductor Interface. Scientific Reports 3 (2013) 1-6.

15. Patel D. K., Kim H.B., Dutta S. D., Ganguly K., Lim K.T.: Carbon Nanotubes-Based Nanomaterials and Their Agricultural and Biotechnological Applications. Materials 13 (2020) 1679.

16. Ahmad M. A., Afandi N. S., Adegoke K. A., Bello O. S.: Optimization and batch studies on adsorption of malachite green dye using rambutan seed activated carbon. Desalination and Water Treatment 57 (2015) 21487-21511.

17. Sen S., Manik N. B.: Effect of Carboxyl-Functionalized Single Walled Carbon Nanotubes on the Interfacial Barrier Height of Malachite Green Dye Based Organic Device. Physics International 10 (2019) 1-7.

18. Sen S., Manik N. B.: Study on the Effect of $8 \mathrm{~nm}$ Size Multi Walled Carbon Nanotubes (MWCNT) on the Barrier Height of Malachite Green (MG) Dye Based Organic Device. International Journal of Advanced Science and Engineering 6 (2020) 23-27.

19. Sen S., Manik N. B.: Effect of Zinc Oxide $(\mathrm{ZnO})$ Nanoparticles on Interfacial Barrier Height and Band Bending of Phenosafranin (PSF) Dye-Based Organic Device. Journal of Electronic Materials 49 (2020) 4647-4652.

20. Chiguvare Z., Parisi J., Dyakonov V.: Current limiting mechanisms in indium-tin-oxide/poly3hexylthiophene/aluminum thin film devices. Journal of Applied Physics 94 (2003) 2440-2448.

21. Harrabi Z., Jomni S., Beji L., Bouazizi A.: Distribution of barrier heights in Au/porous GaAs Schottky diodes from current-voltage-temperature measurements. Physica B 405 (2010) 3745-3750.

22. Al-Ta'ii H. M. J., Amin Y. M., Periasamy V.: Calculation of the Electronic Parameters of an Al/DNA/p-Si Schottky Barrier Diode Influenced by Alpha Radiation. Sensors 15 (2015) 4810-4822. 
23. Sen S., Manik N. B.: Effect of Back Electrode on Trap Energy and Interfacial Barrier Height of Crystal Violet (CV) Dye based Organic Device. Bulletin of Materials Science 43 (2020) 1-4.

24. Selçuk A. B., Ocak S. B., Kahraman G., Selçuk A. H.: Investigation of diode parameters using I-V and $\mathrm{C}-\mathrm{V}$ characteristics of $\mathrm{Al} / \mathrm{maleic}$ anhydride (MA)/p-Si structure. Bulletin of Materials Science 37 (2014) 1717-1724.

25. Yildirim M.: Determination of Contact Parameters of Au/n-Ge Schottky Barrier Diode with Rubrene Interlayer. Journal of Polytechnic 20 (2017) 165-173.

26. Zhang T., Raynaud C., Planson D.: Measure and analysis of 4H-SiC Schottky barrier height with Mo contacts. The European Physical Journal Applied Physics 85 (2019) 10102-1 -10102-9.

27. Norde H.: A modified forward I-V plot for Schottky diodes with high series resistance. Journal of Applied Physics 50 (1979) 5052-5053.

28. Yakuphanoglu F., Shah M., Farooq W. A.: Electrical and Interfacial Properties of p-Si/P3HT Organic-on-Inorganic Junction Barrier. Acta Physica Polonica A 120 (2011) 558-562.

29. Kocyigit A., Yılmaz M., Aydogan Ș., Incekara Ü.: The effect of measurements and layer coating homogeneity of AB on the Al/AB/p-Si devices. Journal of Alloys and Compounds 790 (2019) 388396.

30. Türüt A.: Determination of barrier height temperature coefficient by Norde's method in ideal Co/nGaAs Schottky contacts. Turkish Journal of Physics 36 (2012) 235-244.

31. Fukui K.: Theory of Orientation and Stereo Selection. Springer, Berlin, Heidelberg 1975.

32. Haldar A., Maity S., Manik N. B.: Effect of back electrode on photovoltaic properties of crystalviolet-dye-doped solid-state thin film. Ionics 14 (2008) 427-432. 